\title{
Prevalence of MRSA, ESBL and Carbapenemase Producing Isolates Obtained from Endotracheal and Tracheal Tubes Secretions of ICU Patient at Tertiary Care Centre
}

\author{
Sayyeda Gulsitan Siddique*, Manjushree H. Bhalchandra, Anupama S. Wyawahare, \\ Vishvesh P. Bansal, Jyotsna K. Mishra and S.D. Naik
}

\author{
Department of Microbiology MGM Medical College, Aurangabad, Maharashtra, India \\ *Corresponding author
}

\section{A B S T R A C T}

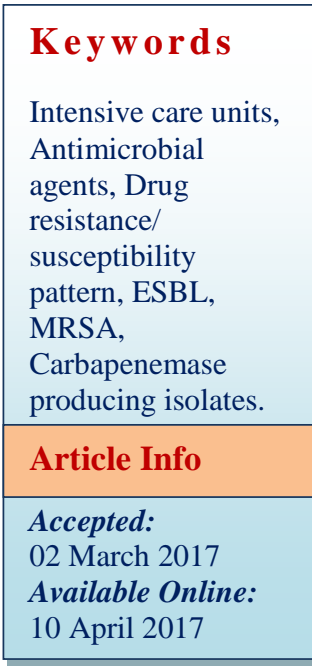

Microbiological infection plays vital role in determining the outcome as well as cost and duration of the hospital stay for patients admitted in ICU setup. Of late nosocomial infections especially ventilator associated pneumonias (VAP) have been recognized to be associated with significant morbidity and mortality in intubated patients. It is of critical importance to do regular surveillance of important pathogen (like Methicillin resistant staphylococcus aureus (MRSA), extended spectrum beta lactamase (ESBL) and carbapenemase producers) and its resistance pattern. Culture and sensitivity patterns if properly determined can greatly improve the outcome of critically ill patients being managed in intensive care units. To find out the prevalence of MRSA, ESBL and carbapenemase producing isolates in endotracheal and tracheostomy tube samples of ICU patients and to know its resistance pattern. In this study we aimed to investigate the pattern of microorganism grown and their sensitivity patterns to propose a proper antibiotic treatment in our hospital ICUs. It was a cross sectional observational study which was carried out over a period of 6 months in the department of microbiology at a tertiary care hospital. Approval of ethical committee was obtained before the study. Total 70 samples consisting of endotracheal tubes and tracheostomy tube secretions received in the department of microbiology for culture and sensitivity from patients admitted in two ICUs (SICU, MICU) were processed as per standard microbiological protocol. Pathogens were identified by vitek 2 compact system. MRSA, ESBL and carbapenemase production was detected by vitek 2 system (Biomerieux) antibioticsusceptibility testing of MRSA, ESBL and carbapenemase producers was done. Antibiotics for testing were chosen as per vitek Id cards. The sensitivity patterns of MRSA, ESBL and carbapenamase producers were analyzed. Out of 70 samples $48(68.57 \%)$ were culture positive in which $41(87.27 \%)$ were monomicrobial and $7(12.72 \%)$ were polymicrobial (2 organisms in 7 samples). Out of 55 isolated organisms 8 were gram positive cocci (14.54\%) and Gram negative organisms were 47 $(85.46 \%)$. Thus total 9 types of organisms were recovered from 48 culture positive samples. Forteen Pseudomonas aeruginosa (25.54\%), 14 Acinetobacter baumanni complex (25.54\%), 11 Klebsiella pneumoniae. (20\%) and 8 Staphylococci (including Staphylocococcus aureus 03 and 05 coagulase negative Staphylococcus (CONS) (14.54\%). Followed by 3 E. coli (5.45\%), 2 Enterobacter aerogens $(3.64 \%), 1$ species each of Serratia marscens, Stenotrophomonas (1.82\%). Out of 8 Staphylococcus (S. aureus and CONS) isolates $5(62.5 \%)$ were MRSA and MRCONS. Most of the MRSA isolates were recovered from the endotracheal tube. Out of all isolates of Klebsiella species and E. coli 8 species were ESBL producers (57.14\%). The maximum ESBL producing isolates were recovered from MICU and were isolated from endotracheal tubes. Isolated MRSA strains were found to be susceptible to vancomycin, linezolid, teicoplanin and rifampicin amongst tested antibiotics. In isolated ESBL producing organisms the highest susceptibility was noted for colistin and tigecycline. The carbapenemase producing isolates $28(59.57 \%)$ showed susceptibility to colistin, amikacin and tigicycline. In present study the prevalence of MRSA, ESBL and carbapenemase producing isolates was $62.5 \%, 57.14 \%, 59.57 \%$ respectively. MRSA isolates were found to be susceptible to antibiotics like vancomycin, rifampicin, linezolid and teicoplanin while ESBL producing isolates were found to be susceptible to antibiotics like imipenem, colistin and tigicycline. Carbapenemase producing isolates were found to be susceptible to antibiotics like colistin, amikacin and tigicycline. Most of MRSA and carbapenemase producers were from SICU and ESBL from MICU. So we need to strengthen our infection control practices so as to reduce these infections. 


\section{Introduction}

Intensive care units are specialized wards in hospitals to offer close monitoring and personalized care to very sick patients in whom there is imminent risk to life. ICUs despite their apparent impact on patient outcome have become high risk areas for health care associated infection. The patient in the ICU has a 5-7 fold higher risk of a health care associated infection compared with the average patient and $20-25 \%$ of all health care associated infection develops in ICUs (Wenzel et al., 1983). Critically ill patients admitted in ICUs are always at higher risk of developing infections with various antibiotic resistant organisms (Like MRSA, ESBL and CARBAPENEMASE producers) due to prolonged hospitalization, serious illness, compromised hosts, usage of invasive devices and procedures, higher rates and longer duration of antibiotic usage and exogenous transmission of bacteria, usually by hospital personnel (Dasgupta et al., 2015; Shanmuga Vadivo et al., 2014). Frequent and indiscriminate use of broad - spectrum antibiotics without culture sensitivity tests results in colonization with multi resistant organisms (Like MRSA, ESBL \& CARBAPENEMASE producers) and consequently in serious infection (Alireza et al.,; Kiran et al., (2015); Dautzenberg et al., 2015). ESBLs were first identified in the early 1980s; since then, ESBLs have been identified worldwide and have been found in a number of different organisms, including Klebsiella pneumoniae, Escherichia coli, Proteus mirabilis and Salmonella spp. The advent of ESBL producers has represented a great threat to the use of many classes of antibiotics, particularly cephalosporins (The microbiological profile of nosocomial infections in the intensive care unit). It is well known that poor outcomes occur when patients with serious infections due to ESBL producing organisms are treated with antibiotics to which the organism is frankly resistant. The mortality rate in such patient is significantly higher than that in patients treated with antibiotics to which the organism is susceptible (The microbiological profile of nosocomial infections in the intensive care unit).

MRSA strains have emerged in the last decade as one of the most important nosocomial pathogen in ICU patients. Infected and colonized patients provide the primary reservoir and transmission is mainly through staff. The risk factors which contribute to MRSA are excessive antibiotic usage, prolonged hospitalization and intravascular catheterization. More than $70 \%$ of Staphylococcus aureus isolated in ICUs is MRSA. Its detection is important for prevention of its transmission as well as for effective treatment of patients (Sista et al., 2004).

Of late carbapenemase producing organisms have emerged as a challenge for treating physicians in intensive care units. Carbapenem resistant organisms especially carbapenem resistant Klebsiella pneumoniae (CRKP) is one of the common bacteria responsible for sepsis in ICUs (Mouloudi et al., 2014). Invasive procedures like central lines and urinary catheters are very important for management of critically ill patients but they are also responsible for life threatening consequences due to severe resistant infections. Antibiotic overuse and misuse partly due to incorrect diagnosis; and irregular consumptions due to wrong prescription or poor compliance all contributes to the worldwide drug resistance among the hospital acquired organisms (Radji et al., 2011).

One of the important nosocomial infection is ventilator associated pneumonia (VAP) seen in patients who have been intubated for mechanical ventilation. Since these patients are already critically ill occurrence of VAP 
seriously jeopardizes the chances of recovery. The mortality rates from VAP vary widely depending upon the demography and status of healthcare in that particular country and have been reported up to 50\% (Baker et al., 1996). Common organisms involved in ventilator associated pneumonia include Gram negative organisms like Pseudomonas aeruginosa, Escherichia coli, Klebsiella pneumoniae and Acinetobacter species, and Gram-positive organisms such as Staphylococcus. Multibacterial infections and changing bacterial infections are also common in patients who have been admitted in ICU for prolonged periods. These changing flora complicate therapy by acquiring drug resistance and altering their sensitivity pattern (Montravers et al., 2015). Therefore updated knowledge of local epidemiological and susceptibility profile is recommended for guiding clinicians regarding empirical choice of antibiotics and has become mandatory along with adequate clinical diagnosis and bacterial confirmation (Aarts et al., 2007). Rapid identification of the bacteria and antimicrobial therapy based upon the culture and sensitivity patterns of the involved organisms is the gold standard treatment of any infection. For this purpose automated bacterial identification systems have been in use since decades.

Only few of them are being used now a day. Some of the rapid identification systems which are currently in use are ATB, MicroScan and VITEk. The newer version of VITEK namely VITEK 2 system (bioMérieux, Marcy l'Etoile, France) is significant as it gives results within 3 hours (Cuziat et al., 1997). This early detection is based upon its mechanism of detection which is florescence based and more sensitive in detecting metabolic changes thereby continually monitoring reactions and in the process provides much faster detection and identification (Gayral et al., 1997).
Aim of our study was to determine the aerobic bacterial profile and antibiotic sensitivity and resistance pattern of isolates from endotracheal secretions in a tertiary care referral centre. Each isolate was identified by using automated VITEK 2 compact system.

\section{Materials and Methods}

This Cross - sectional observational study was conducted after the approval from Ethical Committee. Seventy samples of endotracheal and tracheostomy secretions were included in the study which were received from ICU (MICU, SICU) patients for culture and sensitivity between Jan 2016to June2016 in the Microbiology Laboratory of MGM medical college Aurangabad. Gram's staining of received samples was done to rule out whether it was a colonizer/pathogen using Qscore (Barret Score) (Winn et al., 2006). Received samples were inoculated on 5\%sheep blood agar and MacConkeys agar and incubated overnight at $35^{\circ} \mathrm{C}$. Each growth was gram stained to see whether it was gram positive /gram negative cocci /bacilli (Patricia, 2014). The card is selected. Each organism suspension was prepared from the growth of pure cultures. Bacterial cells were suspended in $2.5 \mathrm{ml}$ of a $0.45 \%$ sodium chloride solution. The suspension used in the VITEK 2 system was adjusted to 0.5 Mcfarland standard by densicheck (Biomeriuex). Isolated organisms were subjected for identification of MRSA, ESBL and CARBAPENEMASE and susceptibility testing was done using Vitek 2 compact system (As per manufacturer's instruction).

\section{Results and Discussion}

The present study was carried out in a tertiary care hospital in Aurangabad. The samples received in the department of Microbiology were processed for culture and sensitivity by using Vitek 2 compact system. It identified 
MRSA, ESBL and CARBAPENEMASE producers were identified with Vitek 2 compact system and recorded by it.

Out of 70 samples received in microbiology lab from the patients admitted in ICUs 15 $(31.42 \%)$ were culture negative and 48 (68.57\%) were culture positive. Upon arrival of the sample in the microbiology lab the relevant history, sign and symptoms of the patient were noted so as to make a correlation with the clinical condition of the patient.

Out of the culture positive cases $41(85.41 \%)$ were monomicrobial and 7 (14.59\%) were polymicrobial (2 organisms in 7 samples). Polymicrobial growth was most commonly associated with those patients who were being treated for diseases requiring prolonged stay in the hospital. Moreover polymicrobial growth was also associated with invasive procedures like central lines or catheterization.

The analysis of the organisms isolated from endotracheal and tracheal tube secretions showed that the commonest organism isolated were Pseudomonas spp, Acinetobacter each (25.54\%) and Klebsiella (20\%)(Table 1).

The analysis of origin of the microbiology sample revealed that the 51 samples were received from endotracheal secretion while 19 samples were from tracheal tube secretions. The presence of predominantly endotracheal samples was probably because of artificial ventilation required in intensive care units (Table 2).

Amongst isolated organisms gram positive cocci were $8(14.54 \%)$ and Gram negative organisms were $47(85.46 \%$ ) (Table 3 ). The analysis of organisms isolated from the ICU patients revealed that out of $8(14.54 \%)$ staphylococcus (aureus and coagulase negative Staphylococci) isolated 5 were
Methicillin resistant Staphylococcus aureus (MRSA) (3) and 2 were methicillin resistant coagulase negative Staphylococci (MRCONS). Out of these 5 samples 3 $(5.45 \%)$ belonged to SICU and 2 (3.63\%) belonged to MICU. So out of all MRSA isolated $60 \%$ belonged to SICU and $40 \%$ belonged to MICU.

Out of the total 5 MRSA, MRCONS isolates, the highest susceptibility was noted for Vancomycin (100\%), Rifampicin and Linezolid (80\% susceptibility for each one of them) followed by Azithromycin (40\%) and erythromycin (20\%).

Regarding isolation of Gram Negative Bacilli (GNB) from samples of ICU patients, Out of isolates of E. coli, and Klebsiella spp. 8 (57.14\%) were ESBL producers.

Out of 3 ESBL producers of E. coli, 2 isolates were obtained from MICU and 1 isolate was found from the samples from SICU and out of 5 ESBL producers of Klebsiella spp., 3 were obtained from MICU and 2 isolates were found from SICU. The final analysis revealed that most of the extended spectrum beta lactase producing organisms was found in Endotracheal tube samples from Medical intensive care unit.

Out of total 8 ESBL producing E. coli and Klebsiella 6 were found in samples from endotracheal tube secretions and 2 were found in tracheostomy tubes.

The final analysis of ESBL producing organism revealed that the highest susceptibility of the ESBL producing organisms was found to be towards colistin, tigicycline followed by imipenem. While ESBL producing E. coli were $66.66 \%$ and Klebsiella were $80 \%$ resistant to piperacillin/tazobactum. Out of the culture positive ESBL isolates of $E$. coli organism the 
highest susceptibility was noted for amikacin $(100 \%)$ followed by colistin (66.66\%), tigicycline $(66.66 \%)$ and imipenem(66\%). Whereas for Klebsiella isolates, the highest susceptibility was noted for colistin (80\%) and tigicycline $(80 \%)$ followed by imipenem $(60 \%)$, levofloxacin and amikacin.

Analysis of carbapenemase producing organisms revealed that Acinetobacter $(42.85 \%)$ was the most common organism producing carbapenemase in various samples received from intensive care units. Out of 14 Acinetobacter $12 \quad(85.71 \%) \quad$ were carbapenemase producer and among 14 $\begin{array}{lll}\text { Pseudomonas } & 8 & (28.57 \%)\end{array}$ carbapenemase producers (Table 9). Likewise carbapenamase producing isolates on susceptibility testing revealed the similar pattern of susceptibility. They are found to be most sensitive to tigicycline, colistin and rifampicin. The susceptibility pattern of Acinetobacter, Pseudomonas, E. coli Klebsiella for colistin is $91.66 \%, 87.5 \%$, $100 \%, 80 \%$ and for tigecycline is $100 \%$,
$87.5 \%, 100 \%$ and $80 \%$ respectively. While none of carbapenemase was susceptible to imipenem. The maximum isolation of carbapenemase producing organisms was from SICU (Table 11).

The analysis of resistance patterns of various organisms depending upon their sample origin revealed that the most of the organisms belonging to both ET and TT were carbapenemase producing followed by ESBL producing and MRSA (Table 12).

The analysis of the data in our study revealed that the most common drug effective for the treatment of MRSA was rifampicin, lizolid and teicoplanin while the ESBL and carbapenemase producing gram negative organisms like E. coli and Klebsiella were found to be susceptible to colistin, tigicyclin and rifampicin and in emergency situation these susceptibility patterns are important while starting empirical antibiotic therapy in ICU patients belonging to our hospital

Table.1 Organisms isolated from ET and TT of patients admitted in ICU

\begin{tabular}{|l|l|l|}
\hline S.No. & Organism & Number $(\%)$ \\
\hline 1 & Staphylococcus aureus & $3(5.45 \%)$ \\
\hline 2 & CONS & $5(9.09 \%)$ \\
\hline 3 & Enterobacter aerogens & $2(3.64 \%)$ \\
\hline 4 & E-coli. & $3(5.45 \%)$ \\
\hline 5 & Klebsiella Spp & $11(20 \%)$ \\
\hline 6 & Stenotrophomonas & $1(1.82 \%)$ \\
\hline 7 & Serratia & $2(3.64 \%)$ \\
\hline 8 & Pseudomonas spp & $14(25.45 \%)$ \\
\hline 9 & Acinetobacter $\operatorname{spp}$ & $14(25.45 \%)$ \\
\hline & Total & $55(100 \%)$ \\
\hline
\end{tabular}


Int.J.Curr.Microbiol.App.Sci (2017) 6(4): 288-299

Table.2 Origin of samples in studied cases

\begin{tabular}{|l|l|}
\hline Sample & No of samples \\
\hline Endotracheal Tube & $51(72.85 \%)$ \\
\hline TT & $19(27.15 \%)$ \\
\hline
\end{tabular}

Table.3 Percentage of GNB and GPC

\begin{tabular}{|l|l|}
\hline GNB & $\mathbf{4 7}(\mathbf{8 5 . 4 6 \%})$ \\
\hline GPC & $8(14.54 \%)$ \\
\hline
\end{tabular}

Table.4 MRSA isolated in different types of ICUs

\begin{tabular}{|ll|}
\hline Location & $\begin{array}{l}\text { Number of MRSA, } \\
\text { MRCONS }\end{array}$ \\
\hline SICU & 3 \\
\hline MICU & 2 \\
\hline
\end{tabular}

Table.5 Susceptibility pattern of MRSA isolates

Erythromycin Azithromycin Levofloxacin Rifampicin Linezolid Teicoplanin Vancomycin

$$
2(40 \%)
$$

None

$4(80 \%)$

$4(80 \%)$

$3(60 \%)$

$5(100 \%)$

Table.6 ESBL producing E. coli and Klebsiella spp. isolated in different ICUs

\begin{tabular}{|l|r|r|}
\hline Type Of ICU & \multicolumn{3}{|c|}{ Klebsiella spp $(\mathbf{n}=5)$} \\
\hline MICU & 2 & 3 \\
\hline SICU & 1 & 2 \\
\hline
\end{tabular}

Table.7 Sample wise distribution of ESBL producing E. coli and Klebsiella spp. isolated in different ICUs

\begin{tabular}{|l|r|r|}
\hline Type Of Specimen & E. coli $(\mathbf{n = 3})$ & \multicolumn{2}{|c|}{ Klebsiella spp (n=5) } \\
\hline Endotracheal Tube Secretions & 2 & 4 \\
\hline TT & 1 & 1 \\
\hline
\end{tabular}


Table.8 Susceptibility pattern of ESBL producing Klebsiella spp. and E. coli

\begin{tabular}{|c|c|c|c|c|c|c|c|}
\hline ESBL & COL & Tig & LE & AK & PIT & CTR & IPM \\
\hline E. coli $(\mathrm{n}=3)$ & 2 & 2 & 2 & 3 & 1 & None & 2 \\
\hline $\begin{array}{c}\text { Klebsiella } \\
\text { Spp }(\mathrm{n}-=5)\end{array}$ & 4 & 4 & 2 & 2 & 1 & 1 & 3 \\
\hline
\end{tabular}

Table.9 Carbapenemase producing organisms

\begin{tabular}{|c|c|c|}
\hline Type of organism & Number & Percentage \% \\
\hline Acinetobacter & $12(42.85 \%)$ & $85.71 \%$ \\
\hline Pseudomonas & $\begin{array}{ll}8 & (28.57 \%)\end{array}$ & $57.14 \%$ \\
\hline Klebsiella & $5 \quad(17.85 \%)$ & $45.45 \%$ \\
\hline E-coli & $1 \quad(3.57 \%)$ & $33.33 \%$ \\
\hline Others & $\begin{array}{ll}2 & (7.14 \%)\end{array}$ & $66.66 \%$ \\
\hline Total & $28(100 \%)$ & \\
\hline
\end{tabular}

Table.10 Susceptibility pattern of carbapenemase producing Acinetobacter, Pseudomonas, Klebsiella spp. and E. coli

\begin{tabular}{|c|c|c|c|c|c|c|c|}
$\begin{array}{c}\text { Carbapenemase Producing } \\
\text { isolates }\end{array}$ & Rif & COL & Tig & LE & AK & \multicolumn{3}{|c|}{ IMP } & CTR \\
\hline Acinetobacter $(\mathbf{n = 1 2})$ & 10 & 11 & 12 & 9 & 8 & None & 1 \\
\hline Pseudomonas $(\mathrm{n}=\mathbf{8})$ & 6 & 7 & 7 & 7 & 6 & None & 2 \\
\hline E. coli $(\mathrm{n}=1)$ & 1 & 1 & 1 & None & 1 & None & None \\
\hline Klebsiella Spp (n-=5) & 4 & 4 & 4 & 3 & 2 & None & 1 \\
\hline & & & & & & & \\
\hline
\end{tabular}

Table.11 Carbapenemase producing organisms isolated in different ICUs

\begin{tabular}{|l|l|}
\hline Type of ICU \\
\hline MICU & 12 \\
\hline SICU & 16 \\
\hline
\end{tabular}


Table.12 Organisms producing carbapenemase/ ESBL/ MRSA

\begin{tabular}{lll}
\hline Sample & \multicolumn{2}{l}{ Organisms producing carbapenemase/ ESBL/ MRSA } \\
\hline ET & Carbapenemase producing & 22 \\
& ESBL & 6 \\
& MRSA & 3 \\
& Carbapenemase producing & 6 \\
TT & ESBL & 2 \\
& MRSA & 2 \\
\hline
\end{tabular}

Figure.1 Culture positive and culture negative samples

\section{Region 1}

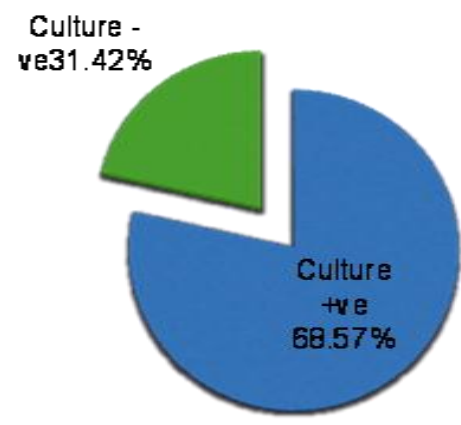

Figure.2 Monomicrobial Vs polymicrobial growth

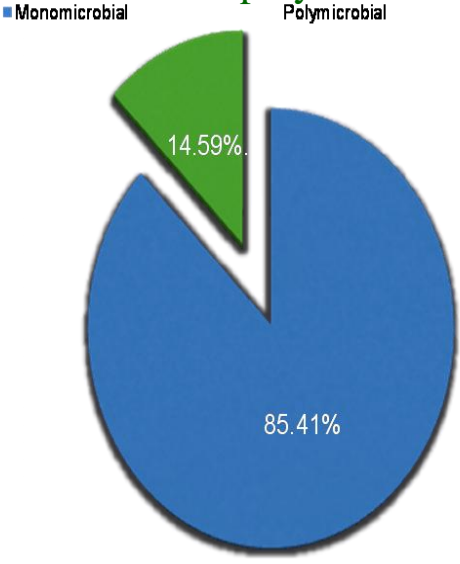

Out of 70 samples $48(68.57 \%)$ were culture positive in which $41(87.27 \%)$ were monomicrobial and $7 \quad(12.72 \%)$ were polymicrobial (2 organisms in 7 samples). 
There were $42(60 \%)$ male and $28(40 \%)$ females. Out of 55 isolated organisms 8 were gram positive cocci $(14.54 \%)$ and Gram negative organisms $47(85.46 \%)$. Thus total 9 types of organisms were recovered from 48 culture positive samples. The culture positivity in Pratirodh Koirala et al., study was higher (90\%) compared to our culture positivity rate of $68.57 \%$, might be due to our hospitals good infection control practices. Our study results of culture positivity is in concordance with results of Ashis Kumar Saha $(59.6 \%)$ and incidence of male positivity was $69.17 \%$, female positivity was $(26.25 \%)$ (Ashis et al., 2016) In our study the predominant organisms isolated were Gram negative organisms $47(85.46 \%)$ from all the ICUs including Pseudomonas spp (25.54\%), Acinetobacter spp (25.54\%), Klebsiella spp. (20\%) and Staphylococci (14.54\%) followed by, E. coli (5.45\%), Enterobacter aerogens (3.64\%), Serratia marscens (3.64\%) and stenotrophomonas $(1.82 \%)$, which matches to a study by Abdulllahi Alireza et al., (2013) study, in their study Gram negative were the most common isolated organisms including Acinetobacter (24.2\%) was the commonest isolated organism from endotracheal aspirates, followed by Pseudomonas (16.7\%) and staphylococci(12\%). A study by Tandia et $a l$. , also matches to our study, the commonest isolated organism was Pseudomonas, followed by staphylococci in their study.

Indian Network for Surveillance of Antimicrobial Resistance (INSAR) group, India conducted a comprehensive research about the prevalence of MRSA across major hospitals and medical institutes of India they found the incidence of MRSA to be $53 \%$ at all India institute of medical sciences, $60 \%$ at Jawahar Lal Nehru institute of postgraduate medical education and research puducherry, $84 \%$ at regional institute of medical sciences imphal. These results were similar to the results of our study in which we found the incidence of MRSA to be $65.5 \%$ (Joshi et al., 2013). Barai et al in Dhaka also had a MRSA prevalence of 77\% (Barai et al., 2010).

Out of the all isolates of MRSA, the highest susceptibility, about $80 \%$, was noted for linezolid and rifampicin. Many studies have concluded similar sensitivity patterns for MRSA sensitivity. Ali khaled et al., (2013) in their study found MRSA to be uniformly sensitive to linezolid. Al-zoubi et al., (2015) conducted a study on the sensitivity patterns of MRSA isolates, they found $85.8 \%$ of the isolated MRSA sensitive to rifampicin. CLSI recommends that all resistant isolates for linezolid should be confirmed by MIC testing. However this was not done in our study. If at all if it would have been done, the susceptibility to linezolid would have been still higher like Kassim et al., in their study, 1103 S. aureus MICs were analyzed and found that susceptibility to linezolid, vancomycin and clindamycin, was comparable between the two guidelines.

The growth and sensitivity patterns of ESBL also revealed these organisms to be sensitive to rifampicin, colistin and tigicycline. Bishara et al., (2005) found ESBL producing E. coli and Klebsiella to be equally susceptible to colistin and etrapenem and $82 \%$ resistant for Klebsiella to Piperacillin /tazobactum. This sensitivity to colistin and resistance pattern of ESBL for Piperacillin /tazobactum (80\%) also matches to our study for ESBL Klebsiella. Hoban et al., (2007) found tigecycline and minocycline to be most active against extended-spectrum beta-lactamase producers and multidrug-resistant Acinetobacter baumannii and these were resistant to cefepime, ceftazidime, ceftriaxone, levofloxacin, and piperacillin-tazobactam.

The carbapenemase producing organisms in our study were found to be susceptible to rifampicin, colistin and tigicycline. These 
findings were in conformity with many studies conducted on the growth and sensitivity patterns of carbapenemase producing organisms. Dheepa muthusamy et al., (2015) found a good number of carbapenem resistant organisms to be sensitive to polymyxins like colistin and polymyxin $\mathrm{B}$. Hence, they recommended that polymyxins like colistin and polymyxin B to be considered as the next drug of choice for carbapenem resistant strains. In this study these isolates were $100 \%$ resistant to Imipenenem this result of imipenem was in concordance with our study. As in our study in Amina Kandeel's study she stated 100\% sensitivity of carbapenemase producers to Colistin followed by tigecycline. But in contrast to this a number of other studies by Soren et al., and Lee et al., have found rifampicin to be highly effective in treating infections caused by Gm-ve ESBL producing organisms (Soren et al., 2015; Lee et al., 2013).

It is concluded in the present study that the prevalence of MRSA, ESBL and Carbapenemase producing isolates was $62.5 \%, 57.14 \%$ and $59.57 \%$ respectively. Most of the MRSA isolates and Carbapenemase producers were recovered from SICU. The maximum ESBL producing isolates were Klebsiella spp. and were recovered from MICU and from endotracheal secretions. So as to reduce the prevalence of these pathogens we need to strengthen the infection control practices in ICUs, screening of health care worker for MRSA nasal carriage and their treatment, to avoid excessive antibiotic usage, prolonged hospitalization and unnecessary intravascular catheterization. MRSA isolates were found to be most sensitive to antibiotics like Vancomycin, Rifampicin, linezolid. ESBL producing organisms were found to be susceptible to drugs like colistin, tigecycline, amikacin and Imipenem, while carbapenemase producing organisms were found to be most susceptible to antibiotics like, colistin, tigicycline and rifampicin. The present results can be used as a guide for choosing an appropriate therapy, particularly when treating suspected MRSA, ESBL and Carbapenemase infections in our ICU patients. These guidelines can decrease morbidity and mortality in patients admitted in ICU.

\section{References}

Aarts, M.A., Granton, J., Cook, D.J., Bohnen, J.M., Marshall, J.C. 2007. Empiric antimicrobialtherapy in critical illness: results of a surgical infection society survey. Surg. Infect., (Larchmt). 8(3): 329-36.

Ali Khalid, Javaid Usman, Umaira Faiz, Fatima Kaleem, Afreenish Hassan, Maria Omair. In-vitro susceptibility of linezolid against methicillin resistant Staphylococcus aureus at a tertiary care hospital in Pakistan, J. Microbiol. Infect. Dis., 3(4): 203-206.

Alireza Abdollahi, Saeed Shoar, Nasrin Shoar. 2013. Microorganisms colonization and their antibiotic resistance pattern in orotracheal tube. Iran J. Microbiol., 5(2):102-7

Al-Zoubi, M.S., Al-Tayyar, I.A., Hussein, E., Jabali, A.A., Khudairat, S. 2015. Antimicrobial susceptibilitypattern of Staphylococcus aureus isolated from clinical specimens in Northern area of Jordan. Iranian J. Microbiol., 7(5): 265272.

Amina Kandeel. 2015. Epidemiology of carbapenemase producing Enterobacteriaceae in general hospital, $J$. Microbiol. Infect. Dis., 5(2): 57-62.

Ashis Kumar Saha, Suman Nandi, Payodhi Dhar Prevalence of Bacterial Isolates in Endotracheal Tube According to Culture and Sensitivity in Patients of Intensive Care Unit of A Tertiary Medical College and Hospital, Kolkata, West Bengal Int. J. 
Contemporary Med. Res., Volume 3, Issue 6.

Baker, A.M., J.W. Meredith, and E.F. Haponik. 1996. Pneumonia in intubated trauma patients. Microbiology and outcomes. Am. J. Respir. Crit. Care Med., 153: 343349.

Barai, L., Fatema, K., Haq, J.A., et al. 2010. Bacterial profile and their antimicrobial resistance pattern in an intensive care unit of a tertiary care hospital of Dhaka. Ibrahim Med. Coll. J., 4(2): 66-9.

Bishara, J., Livne, G., Ashkenazi, S., Levy, I., Pitlik, S., Ofir, O., Lev, B., Samra, Z. 2005. Antibacterial susceptibility of extended-spectrum beta-lactamaseproducing Klebsiella pneumoniae and Escherichia coli. Isr. Med. Assoc. J., 7(5): 298-301.

Chi, S.Y., Kim, T.O., Park, C.W., et al. 2012. Bacterial Pathogens of Ventilator Associated Pneumonia in a Tertiary Referral Hospital. Tuberculosis and Respiratory Dis., 73(1): 32-37.

Cuziat, R., S. Cagnès, R. Cogne, M. Desmonceaux, M. Guicherd, and D. Monget. 1997. Influence of isolation media on the identification of gramnegative rods using the Vitek II system, abstr. P258. Clin. Microbiol. Infect., 3(Suppl. 2): 54.

Dasgupta, S., Das, S., Chawan, N.S., Hazra, A. 2015. Nosocomial infections in the intensive care unit: Incidence, risk factors, outcome and associated pathogens in a public tertiary teaching hospital of Eastern India. Indian Journal of Critical Care Medicine: Peerreviewed, Official Publication of Indian Society of Critical Care Med., 19(1):1420.

Dautzenberg, M.J., Wekesa, A.N., Gniadkowski, M., Antoniadou, A., Giamarellou, H., Petrikkos, G.L., Skiada, A., Brun-Buisson, C., Bonten, M.J., Derde, L.P. 2015. Mastering hospital Antimicrobial Resistance in Europe Work Package 3 Study Team. The association between colonization with carbapenemase producing enterobacteriaceae and overall ICU mortality: an observational cohort study. Crit Care Med., 43(6): 1170-7.

Gayral, J.P., R. Robinson, and D. Sandstedt. 1997. A new integrated system for microbiological testing: the Vitek II system, abstr. P254. Clin. Microbiol. Infect., 3(Suppl. 2):53.

Hoban, D.J., Bouchillon, S.K., Dowzicky, M.J. 2007. Antimicrobial susceptibility ofextended-spectrum beta-lactamase producers and multidrug-resistant Acinetobacter baumannii throughout the United States and comparative in vitro activity of tigecycline, a new glycylcycline antimicrobial. Diagn. Microbiol. Infect. Dis., 57(4): 423-8.

Joshi, S., Ray, P., et al. 2013. Indian Network for Surveillance of Antimicrobial Resistance (INSAR) group, India, Methicillin resistant Staphylococcus aureus (MRSA) in India: Prevalence \& susceptibility pattern. The Indian J. Med. Res., 137(2): 363-369.

Kassim, A., Omuse, G., Premji, Z., Revathi, G. 2016. Comparison of Clinical Laboratory Standards Institute and European Committee on Antimicrobial Susceptibility Testing guidelines for the interpretation of antibiotic susceptibility at a University teaching hospital in Nairobi, Kenya: a cross-sectional study. Annals of Clin. Microbiol. Antimicrobials, 15: 21.

Kiran Tandia, J.L. Wadhwani, Manuj Sharma. 2015. A clinical study of Pattern of Microbiological Colonization of Endotracheal Tube Aspirate on Mechanically Ventilated Patients. IJSR, 4(11): $785-787$.

Lee, C.R., Cho, I.H., Jeong, B.C., Lee, S.H. 2013. Strategies to Minimize Antibiotic Resistance. Int. J. Environ. Res. Public Health, 10(9): 4274-4305.

Montravers, P., Dufour, G., Guglielminotti, J., et al. 2015. Dynamic changes of microbial flora and therapeutic consequences in persistent peritonitis. 
Critical Care, 19(1): 70. doi:10.1186/s13054-015-0789-9.

Mouloudi, E., Massa, E., Papadopoulos, S., Iosifidis, E., Roilides, I., Theodoridou, T., Piperidou, M., Orphanou, A., Passakiotou, M., Imvrios, G., Fouzas, I., Papanikolaou, V., Gritsi-Gerogianni, N. 2014. Bloodstream infections caused by carbapenemase-producing Klebsiella pneumoniae among intensive care unit patients after orthotopic liver transplantation: risk factors for infection and impact of resistance on outcomes. Transplant Proc., 46(9): 3216-8.

Muthusamy, D., Sudhishnaa, S., Boppe, A. 2016. Invitro Activities of Polymyxins and Rifampicin against Carbapenem Resistant Acinetobacter baumannii at a Tertiary Care Hospital from South India. J. Clin. Diag. Res. JCDR, 10(9): DC15DC18.

Patricia, M.T. 2014. Bailey and Scott's diagnostic microbiology. $13^{\text {th }}$ ed. China: Elsevier Mosby.

Pratirodh Koirala, Dwij Raj Bhatta, Prakash Ghimire, Bharat Mani Pokhrel, Upendra Devkota. Bacteriological Profile of Tracheal Aspirates of the Patients Attending a Neuro-Hospital of Nepal. Int. J. Life Sci., 4: 60-65.

Radji, M., Fauziah, S., Aribinuko, N. 2011. Antibiotic sensitivity pattern of bacterial pathogens in the intensive care unit of Fatmawati Hospital, Indonesia. Asian Pacific J. Trop. Biomed., 1(1): 39-42. doi:10.1016/S2221-1691(11)60065-8.
Shanmuga Vadivo, N., Priya Santharam, L. Sudha, G. Kalaiselvi, B.K. Padmavathi, B. Usha, Amar Kumar, Nitesh kumar Jaiswal. 2014. Dynamic Bacterial profile of Endotracheal Aspirates and its sensitivity patterns -A cause of concern Int. J. Cur. Res. Rev., /Vol 06 (10) Page 112.

Sista, R.R., Oda, G., Barr, J. 2004. Methicillinresistant Staphylococcus aureusinfections in ICU patients. Anesthesiol. Clin. North America, 22(3): 405-35.

Soren, O., Brinch, K.S., Patel, D., et al. 2015. Antimicrobial Peptide Novicidin Synergizes with Rifampin, Ceftriaxone, and Ceftazidime against AntibioticResistant Enterobacteriaceae In Vitro. Antimicrobial Agents and Chemother., 59(10): 6233-6240.

The microbiological profile of nosocomial infections in the intensive care unit. $J$. Clin. Diag. Res., 4: 3109-311.

Wenzel, R.P., Thompson, R.L., Landry, S.M., Russell, B.S., Miller, P.J., Ponce de Leon, S., Miller, G.B. Jr. 1983. Hospitalacquired infections in intensive care unit patients: an overview with emphasis on epidemics. Infect Control, 4(5): 371-5.

Winn, W., Jr, Allen, .S, Janda, W., Koneman, E., Procop, G., Schreckenberger, P., Woods, G., editors. Koneman's Color Atlas and Textbook of Diagnostic Microbiology. $6^{\text {th }}$ Edition. United States of America: Lippincott Williams and Wilkins.

\section{How to cite this article:}

Sayyeda Gulsitan Siddique, Manjushree H. Bhalchandra, Anupama S. Wyawahare, Vishvesh P. Bansal, Jyotsna K. Mishra and Naik, S.D. 2017. Prevalence of MRSA, ESBL and Carbapenemase Producing Isolates Obtained from Endotracheal and Tracheal Tubes Secretions of ICU Patient at Tertiary Care Centre. Int.J.Curr.Microbiol.App.Sci. 6(4): 288-299. doi: https://doi.org/10.20546/ijcmas.2017.604.032 